\title{
Clostridium bolteae
}

National Cancer Institute

\section{Source}

National Cancer Institute. Clostridium bolteae. NCI Thesaurus. Code C114250.

A species of oblig ately anaerobic, Gram positive, rod shaped bacteria assigned to the phylum Firmicutes. This bacteria is non-motile, non-spore forming, negative for indole, lecithinase, lipase, catalase and urease, does not hydrolyze esculin or gelatin, does not reduce nitrate, and ferments carbohydrate to produce acetic acid and lactic acid. C. bolteae is a part of the normal human intestinal microflora, but may also be involved in the development of autism and bacteremia. 